\title{
Eugène Houdry (1892-1962) : \\ Une personnalité majeure en terme d'innovation industrielle
}

Jacques BREYSSE*

\section{Résumé}

Eugène Houdry (1892-1962) a marqué durablement le monde du raffinage du pétrole dans les années 1930 en développant un procédé catalytique de craquage des huiles lourdes. C'était l'aboutissement de recherches débutées une décennie auparavant.

Dès 1922, Houdry, ingénieur Arts et Métiers (Chalons) et passionné d'automobiles, était invité à participer à des recherches pour produire des carburants à partir de résidus hydrocarbonés à l'instigation du gouvernement français. Ces travaux portèrent d'abord sur le craquage catalytique des vapeurs obtenues par distillation du lignite, pour lequel une installation industrielle fut construite dans le Gard en 1929 mais qui n'eut pas de suites. À partir de 1927, l'étude du craquage catalytique des coupes lourdes pétrolières, le conduisit à développer la recherche de catalyseurs, associée à leur mise en œuvre en terme de procédés. Il conçut alors un procédé industriel complet de craquage de ces coupes lourdes. Mais c'est aux ÉtatsUnis qu'il put développer ce procédé à l'échelle pilote à partir de 1931. Une première unité industrielle démarra en 1937 avec succès, suivie de beaucoup d'autres. Cette production d'essence à haut indice d'octane eut un impact important sur le déroulement de la deuxième guerre mondiale en termes d'approvisionnement en carburant pour avions.

Après la guerre, tout en gardant des liens avec la France, Houdry s'intéressa à de nombreux autres domaines aux États-Unis dont il est devenu citoyen américain en 1941, et où il est encore honoré aujourd'hui (National Inventors Hall of Fame).

\section{Mots-clés}

Houdry Eugène. Craquage catalytique. Lignite. Coupes lourdes de pétrole. Indice d'octane.

\footnotetext{
Abstract petroleum-refining world in the early 1930s by developing a new process for

* Jacques BREYSSE, SFGP, Groupe d'Histoire de la Chimie (GHC).
}

Eugène Houdry (1892-1962) had a considerable impact on the 
the preparation of high-octane rating gasoline by catalytic cracking of crude oil petroleum. This was the culmination of research work started at the beginning of the previous decade.

In 1922, Houdry, a mechanical engineering and amateur of automobiles, was invited to take part in research on the production of synthetic fuels from coal tar at the instigation of the French government. This work first focused on the catalytic cracking of lignite, and a demonstration plant was built in the South of France in 1929, but without any enduring legacy. From 1927 he also tackled the treatment of fuel and heavy oils with the same objective. In this context he developed new catalysts, using a rigorous catalyst selection procedure, and numerous innovative processes. Finally, he designed a global process for using catalysts to crack heavy oil. This process was first developed in the United States in 1931, and a first successful industrial plant began production in 1937. This allowed an increase in the high-octane rating of the gasoline supply available for airplanes during the Second World War.

Houdry took US citizenship in 1941 but maintained close ties with France. He received numerous awards during his career in the United States, where his memory is still honoured today (National Inventors Hall of Fame).

\section{Keywords}

Houdry Eugène. Catalytic cracking. Lignite. Heavy crude oil. High-octane rating.

\section{Introduction}

Eugène Jules Houdry est né le 17 avril 1892 à Domont (Val d'Oise aujourd'hui). Il était le fils de Jules Houdry, serrurier, et de Émilie Thaïs Julie Lemaire. Il eut un frère, Charles Émile, décédé à l'âge de deux ans (1894-1896). Son père était issu d'une famille nombreuse de huit enfants, dont quatre des garçons furent serruriers à l'image de leur père. Il fait ses études secondaires au Collège Turgot à Paris et prépare le concours des «Arts et Métiers » qu'il réussit en 1908. Il intègre l'École de Châlons-sur-Marne où il fait de très brillantes études (major de sa promotion chaque année) et il a une activité extrascolaire notable, en particulier dans l'équipe de football championne de France scolaire. 
À sa sortie en 1911, il rejoint l'entreprise familiale spécialisée en serrurerie et charpente métallique, installée à Juvisy (Essonne aujourd'hui). Mobilisé en 1914, il est incorporé dans l'artillerie, puis dans les chars, où il est nommé lieutenant. Grièvement blessé à Juvincourt en Lorraine (mais heureusement sans conséquence à long terme), il est décoré de la Croix de guerre. En 1918, à la fin de la guerre, il rejoint l'entreprise familiale et s'associe à son père dans l'entreprise Houdry et Fils deux ans plus tard. Dans l'immédiat aprèsguerre, passionné d'automobiles, il ajoute aux activités de l'entreprise, la fabrication d'accessoires automobiles, puis fonde la même année, la Manufacture générale de ressorts dont le siège est au 1 avenue de Villars à Paris, avec des usines à Paris et à Beauchamp dans le Val d'Oise. Il possède alors une Bugatti et visite les États-Unis ou il aurait assisté à la célèbre course des 500 miles d'Indianapolis ${ }^{1}$. Il a alors de nombreux contacts avec les techniciens du pétrole et est rapidement sensibilisé aux problèmes de carburants, que ce soit au niveau qualité (autoallumage) qu'au niveau quantité ${ }^{2}$. Enfin, il se marie le $1^{\text {er }}$ juillet 1922 à Paris avec Geneviève Quilleret, dont il aura deux enfants, Pierre (1924-2004) et Jacques (1926-2012) ${ }^{3}$.

1 Palucka, Tim, «The Wizard of Octane : Eugène Houdry », Invention \& Technology, 20/3, (2005).

2 En 1921, il n'y a en France qu'une très modeste production de pétrole à Pechelbronn, provenant de la mise sous séquestre d'une société allemande qui procédait à cette extraction avant la guerre. Une raffinerie sera construite à proximité, à Merkwiller, en 1924-1925. La raffinerie qui existait à Courchelettes près de Douai, complètement détruite durant la guerre, ne sera remise en service qu'en 1927.

${ }^{3}$ En matière de sources historiographiques sur Eugène Houdry, il faut d'abord mentionner le contenu des conférences de A. Joseph et de E. Houdry lui même sur l' «Historique du cracking catalytique dans l'industrie du pétrole», Bulletin de l'Association française des techniciens du pétrole (1956), p. 179-202 pour Joseph et 


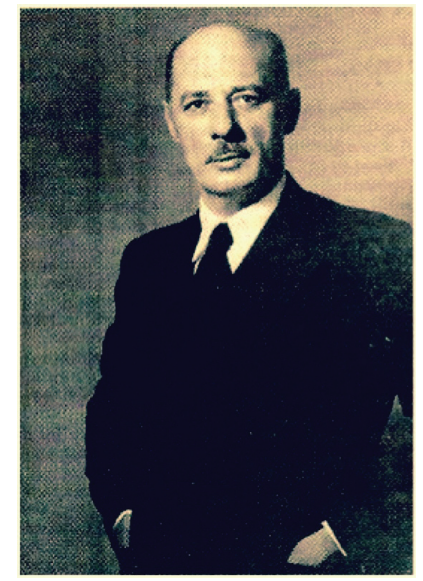

Fig. 1. Eugène Houdry (1960)

\section{Le traitement du lignite ${ }^{4}$ pour obtenir du carburant automobile}

En 1922, la légende veut qu'un coureur automobile, « a fellow racecar driver ", ait montré à Houdry une bouteille de carburant obtenu à partir de lignite par un pharmacien de Nice, E.A. Prudhomme. Au cours de la visite qu'Houdry fait à ce dernier, Prudhomme lui montre une petite installation de laboratoire incluant le chauffage du lignite et le traitement des vapeurs dans un appareil

p. 203-212 pour Houdry. Sur ses apports techniques, voir en particulier Oblad Alex, " The contribution of Eugene Houdry to the development of catalytic cracking ", in Davis Burtron H. et Hettinger William P. (eds), Heterogeneous catalysis: selected American histories, ACS, Washington, 1983, p. 61-75. Enfin on peut mentionner trois articles généraux, ceux de C.G. Moseley (1984), « Eugène Houdry, catalytic cracking and World War II aviation gazoline », Journal of Chemical Education, 61, 65-66 ; De Mills Alex G., "Catalysis: the craft according to Houdry », CHEMTECH, 16, 72-75 (Feb. 1986), et celui de McEvoy Jim, "Citizen Houdry", CHEMTECH, 26, 6-10 (Feb. 1996).

${ }^{4}$ Le lignite est un combustible solide hydrocarboné brun ou noir, dont la teneur en carbone est selon les gisements, entre 15 et 45\%. Il contient aussi de l'eau (5 à 20\%), $\mathrm{du}$ soufre (potentiellement jusqu'à 10\%), des matières volatiles (20 à $40 \%$ en moyenne) et des «cendres ». Voir par exemple à ce sujet Baud Paul, Traité de chimie industrielle. T. 1. Grande Industrie chimique, Masson, Paris, $1951\left(4^{\mathrm{e}}\right.$ édition), p. 370. 
(une colonne ?) contenant du nickel et du cobalt, et permettant l'obtention de quelques gouttes de carburant par heure ! Rentré à Paris, il fait part à des amis de ce qu'il avait vu à Nice puis, malgré la « rusticité » de l'installation et les limites de la démonstration, il constitue avec un certain nombre d'entre eux un «syndicat» chargé d'étudier le procédé proposé par Prudhomme. Houdry organise alors en février 1923 :

"Une mission composée d'un expert officiel et de deux professeurs de faculté accompagnés chacun d'un assistant. L'appareillage utilisé comportait un gazogène à injection de vapeur d'eau, un "scrubber" ${ }^{5}$, un épurateur à "masse de Laming $^{6}$ " et trois catalyseurs successifs dont la composition était à cette époque conservée secrète par Prudhomme. Plusieurs essais contrôlés furent effectués ; on obtint de l'essence paraissant sensiblement analogue à celle $d u$ commerce, mais pendant une période très brève... Les personnalités présentes reconnurent que la qualité du carburant était satisfaisante, mais elles ne purent expliquer les réactions qui avaient lieu. La courte durée de fonctionnement de l'appareil avait malheureusement rendu impossible toute étude quantitative $)^{7}$.

À ce stade, on comprend un peu les hésitations d'Houdry : fallait-il poursuivre ? Il va solliciter de nombreux conseils. Malgré des avis d'experts parfois très négatifs $^{8}$, il décide de poursuivre l'aventure.

\footnotetext{
${ }^{5}$ Scrubber : système (colonne, tour) permettant de séparer des particules (liquides, solides) du gaz dans lequel elles sont entraînées.

${ }^{6}$ Mélanges d'oxydes de fer, de sciure de bois et/ou de sulfate ferreux et de chaux éteinte. Voir Baud P., op. cit., note 4, p. 465 [pour éliminer les composés sulfurés].

${ }^{7}$ Joseph A., op. cit., note 3, p.182.

${ }^{8}$ Palucka, T., op.cit., note 1.
} 
Avec le soutien d'un certain nombre de personnes ${ }^{9}$, il fonde la même année la Société anonyme française pour la fabrication des essences et pétrole avec l'objectif de monter une installation pilote pour étudier le procédé. Il semble bien toutefois qu'il y ait eu un argument décisif qui explique sa décision, à savoir le fait qu'il ait appris que des Italiens s'intéressaient au procédé de Prudhomme et s'apprêtaient à monter eux aussi une unité semi-industrielle... Il décide alors de construire une unité-pilote à Beauchamp, sur les terrains qui jouxtent l'usine de ressorts. Des essais vont démarrer dès mai 1923, sur un procédé dont le principe est identique à celui de Nice.

Au départ, dans l'installation de Prudhomme, il n'est fait état que d'un «gazogène à injection de vapeur» pour la production des gaz à traiter. C'est bien ce qui va être installé à Beauchamp, en l'absence de distillation du lignite, mais les essais ne vont donner aucun résultat ${ }^{10}$ :

"C'est en vain que pendant des journées et des nuits consécutives, Houdry attendit l'arrivée de l'essence dans le séparateur. Il ne récolta que de l'eau et il dut abandonner sa tentative $》$.

Houdry contacte alors ses «amis » italiens qui ont construit une installation similaire. Comme lui, ils n'ont rien obtenu. En concertation avec eux, après «plusieurs mois de travail », il conclut qu'il n'était pas possible dans leurs conditions opératoires de transformer le mélange $\mathrm{CO}+\mathrm{H}_{2}$ en hydrocarbures et que le peu

\footnotetext{
${ }^{9}$ Très exactement six cents, selon T. Palucka, op. cit., note 1 . Houdry conservera longtemps leur soutien, y compris dans les épreuves de son aventure industrielle, jusqu'à ses débuts aux États-Unis.

${ }^{10}$ Joseph A, op. cit., note 3, voir p. 183.
} 
d'essence produite initialement à Nice provenait de l'hydrogénation des goudrons légers non retenus par un scrubber imparfait ${ }^{11}$ !

Après avoir peut-être songé à travailler avec ses correspondants italiens sur leur installation, il décide de relancer des essais à Beauchamp en mai 1924. Cette fois l'objectif n'est plus de séparer les goudrons, mais au contraire de favoriser leur production. Il met donc en place une distillation du lignite ${ }^{12}$ en présence de vapeur d'eau et de gaz réducteurs (hydrogène...). Il supprime le scrubber, maintient le passage des vapeurs sur une " masse de Laming » pour les désulfurer, et réalise un traitement catalytique dans trois tubes contenant successivement du nickel, du nickel/vanadium et du nickel/cobalt (températures entre 180 et $300^{\circ} \mathrm{C}$ )

Les premiers résultats sont, cette fois, positifs avec une productivité régulière mais très faible, l'équivalent de 43 litres d'essence/tonne de lignite (à $23 \%$ d'eau). Ils sont néanmoins suffisamment encourageants pour qu'Houdry décide de poursuivre les études de laboratoire et de monter une installation semi-industrielle. Il fait aussi appel à un expert pour valider et expliquer les résultats obtenus. Ce dernier, dont le nom ne nous est pas connu, conclura en ces termes ${ }^{13}$ :

\footnotetext{
${ }^{11}$ Scrubber : voir note 5. On peut penser que l'idée de départ de Prudhomme était effectivement de faire la synthèse d'hydrocarbures à partir du "gaz de synthèse » $\mathrm{CO}+\mathrm{H}_{2}$. L'idée était « dans l'air », car c'est à cette même époque (1923) que Franz Fischer et Hanz Tropsch vont commencer à breveter des procédés de synthèse organique à partir de $\mathrm{CO}+\mathrm{H}_{2}$. La synthèse d'essence par cette voie $\left(250 / 300^{\circ} \mathrm{C}\right.$, catalyse à base d'oxydes de fer) va être développée et perfectionnée par eux à la fin des années 1920 et donner lieu à d'importantes installations industrielles en Allemagne dans les années 1930.

${ }^{12}$ Sur l'aspect distillation, voir Baud P., op. cit., note 4, p. 388.

${ }^{13}$ Joseph A., op. cit., note 3, p. 185.
} 
"que l'explication la plus plausible... est la mise en ouvre d'hydrogénations successives avec cyclisation et rupture des chaines latérales, permettant aux produits de passer de l'état de composés hydroaromatiques à l'état de composés aromatiques stables ».

À Beauchamp, Houdry décide de passer à l'échelle semi-industrielle ; le 8 mai 1925 il fait une demande d'autorisation d'installer et exploiter un «laboratoire industriel comportant les industries classées ciaprès...» (Voir Annexe: Demande d'autorisation d'Houdry pour Beauchamp, p. 1).

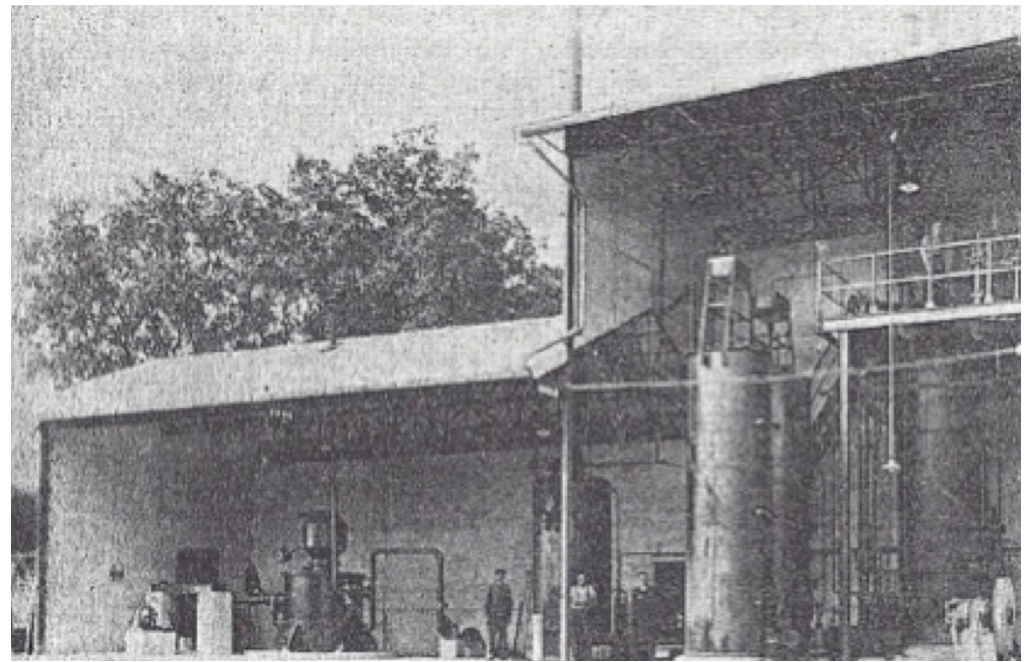

Fig. 2. Installation de la fabrication de gaz à l'eau à Beauchamp

L'installation (base $1 \mathrm{t} / \mathrm{j}$ ) est démarrée en juin 1925, mais très vite elle va donner lieu à de gros problèmes de procédé, à savoir la polymérisation des goudrons vaporisés au niveau de la distillation en raison d'une température trop élevée, la déstructuration des supports de catalyseurs lors de leur «décokage» par soufflage d'air, et enfin une désulfuration encore insuffisante. 
De la fin 1925 au $1^{\text {er }}$ semestre 1926, sont menées diverses études technologiques, notamment pour trouver la bonne technique de distillation, aspect capital du procédé. Des progrès importants vont être faits dans les trois domaines, en particulier pour la distillation, sans être toutefois décisifs ! Par ailleurs, «à l'automne 1926, Houdry avait dû se séparer avec fracas de l'inventeur », comme le dit Joseph en parlant de Prudhomme. L'article paru en 1939 dans la revue Fortune est un peu plus explicite : Prudhomme aurait été surpris en train d'ajouter du gazole à des goudrons (?) pour favoriser leur manipulation lors d'une collaboration avec des clients belges ! En fait, toujours selon l'article, Houdry rompit à cette occasion avec Prudhomme surtout en raison de l'approche de ce dernier qu'il jugeait trop empirique ${ }^{14}$.

\section{Développement industriel du procédé}

Après une nouvelle période d'hésitation, mais aussi des encouragements - et des financements complémentaires - de différents côtés, entre autres de la commission des mines de la Chambre et (probablement) de l'Office national des combustibles liquides ${ }^{15}$, Houdry décide de poursuivre les études de développement, en s'attachant $\grave{a}^{16}$ :

\footnotetext{
14 Collectif, «Monsieur Houdry's Invention », Fortune, 19/2, 56-57, 127-140 (1939), voir p. 130.

${ }^{15}$ L'Office national des combustibles liquides a été créé en janvier 1925 dans le cadre du ministère du Commerce et de l'industrie, avec entre autres pour attributions : «À coordonner et encourager éventuellement par le moyen de subvention ou de primes, les recherches pouvant aboutir à la découverte de pétrole et à la mise au point de carburants de remplacement $»$.
}

${ }^{16}$ Joseph A., op.cit., note 3, p. 188-189. 
"L'étude de la désulfuration, l'étude de la distillation à basse température sur des fours continus de faible capacité, la recherche de catalyseurs appropriés ».

Joseph précise :

"C'est alors que commença une période fort difficile, car un effort intense et permanent, de jour et de nuit, fut effectué pendant les années 1927, 1928, 1929 et 1930. Il ne fut plus question de repos. À côté des laboratoires fut installé un messhôtel dans lequel Houdry et ses collaborateurs avaient leur chambre pour se reposer entre les essais sans se soucier ni de la course du soleil, ni du calendrier $»^{17}$.

Joseph détaille aussi les études de développement et les appareillages utilisés dans l'installation semi-industrielle. En particulier, il mentionne le problème du support des catalyseurs avec d'abord l'abandon de la pierre ponce au profit d'un mélange de kaolin et de terres d'infusoires, puis courant 1927, le développement d'un nouveau support composé de «terre de mussidan », un liant à base de mazout lourd et de sciure de bois (sapin).

Par ailleurs les études de désulfuration vont être très poussées (les lignites étant fortement sulfurés), ainsi que celles de la régénération des catalyseurs (désulfuration, décokage), dont les résultats positifs vont s'avérer d'une grande importance par la suite pour le traitement des gazoles.

L'ensemble des résultats obtenus paraissant industrialisables, Houdry décide d'entreprendre, en février 1927, l'étude d'un installation industrielle sur le carreau des mines de lignite de Saint-Paulet-de-

\footnotetext{
${ }^{17}$ Voir à ce sujet l'article dédié aux souvenirs de Jean Bureau (Saint-Gobain) dans ses contacts avec Houdry, où il détaille une semaine de travail à Beauchamp durant cette période. Bureau Jean, « Notes sur les premiers essais de craquage catalytique par le procédé Houdry : souvenirs de Jean Bureau », in «Aperçu des travaux de Houdry sur le craquage catalytique », Étienne André, L'Actualité chimique (mai 1976), p. 18-22.
} 
Caisson (Gard) dont il était devenu propriétaire l'année précédente. Le 5 août suivant, agissant au nom de la Société des essences et des pétroles de Saint-Julien-de-Peyrolas, Houdry demande à la préfecture du Gard l'autorisation de construction d'une usine de production d'hydrocarbures par distillation des lignites provenant de la mine de Saint-Paulet-de-Caisson. Une enquête d'utilité publique, ouverte le 4 octobre, se déroulera du 10 octobre au 8 novembre 1927. Le Conseil municipal étudie le projet début 1928 et pose un certain nombre de questions auxquelles Houdry répond dans une lettre du 20 juillet 1928. Finalement, moyennant un certain nombre de prescriptions particulières, le Conseil départemental de l'hygiène donne son accord le 28 juillet 1928 .

Houdry n'a pas attendu cet accord pour commencer les études d'implantation dès septembre 1927. Ses premiers objectifs ont été de trouver un four de distillation industriel basse température convenable et d'augmenter la production de lignite de la mine. Le four allemand choisi est capable de traiter $60 \mathrm{t} / \mathrm{j}$ de lignite à 15\% d'eau. La construction commence en avril 1928 et le four mis en route le juin $1929^{18}$. Cependant ce four ne donne pas les résultats escomptés, au mieux $50 \mathrm{t} / \mathrm{j}$, avec un rendement en goudrons initial de $65 \%$ (au lieu de 90 !) qui baisse régulièrement au cours du temps. De plus des difficultés apparaissent au niveau du traitement des dérivés soufrés et de leur transformation en sulfites. L'installation est arrêtée en février 1930 après sept mois de fonctionnement ! L'essence obtenue était convenable, mais contenait encore $3,5 \%$ de soufre sous forme de

\footnotetext{
18 Mandin Yves et Mercier Serge, Mines et usines de Saint Paulet-de-Caisson, Chapitre «Usine de production d'hydrocarbures », 2001, p. 71-115, Archives St Paulet-de-Caisson. Voir p. 92-94.
} 
thiophène. Houdry n'obtint pas l'aide financière qu'il avait sollicitée auprès de l'Office national des combustibles liquides et décida donc d'arrêter progressivement l'usine, qui sera fermée le 15 juin 1930.

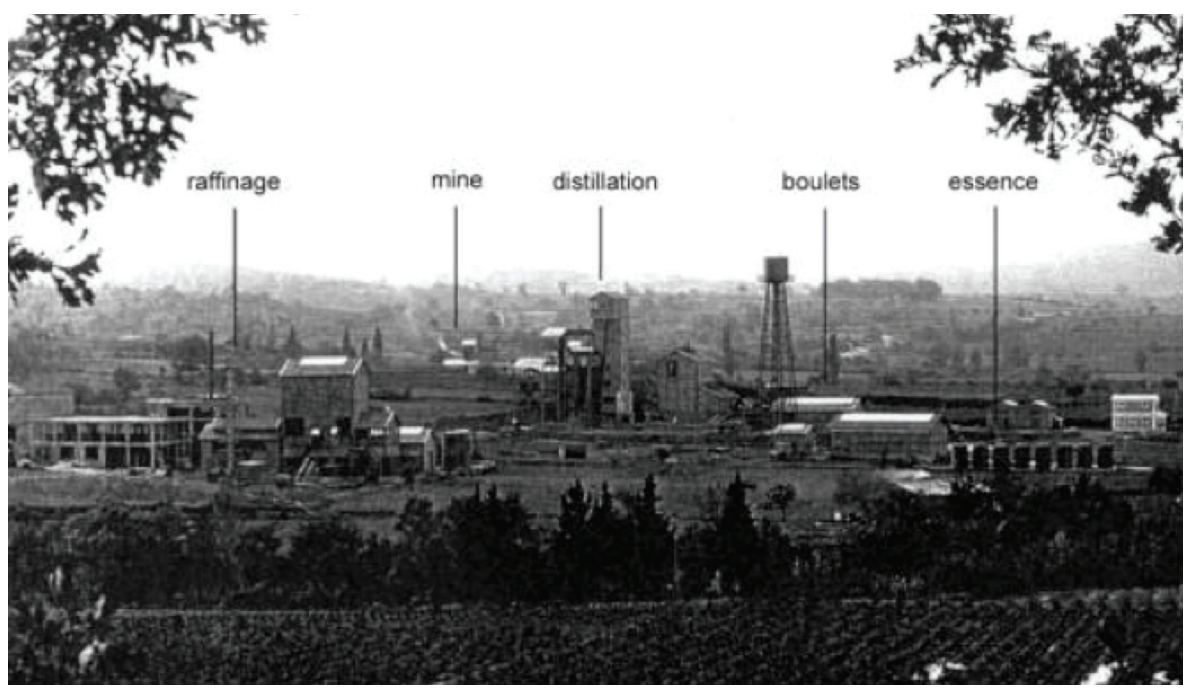

Fig. 2. Usine de Saint-Paulet-de-Caisson

\section{Le traitement des gazoles}

Houdry a commencé à s'intéresser au traitement catalytique des gazoles dès 1927. Les essences obtenues par distillation des pétroles bruts (jusqu'à 20/25\% de la charge initiale dans les cas les plus favorables) étaient à l'époque la source majoritaire de carburant. Mais, il fallait aussi différencier ici les essences légères à indice d'octane $^{19}$ convenable, des essences lourdes où cet indice est beaucoup

\footnotetext{
${ }^{19}$ Dans le cas des moteurs à explosion, le taux de compression, qui conditionne les performances du moteur, peut être limité par les problèmes d'autoallumage ( « cliquetis »») qui réduisent la puissance et peuvent causer des dégâts mécaniques importants. Un moteur qui cliquette signifie que le « niveau d'octane » du carburant est insuffisant. Le niveau d'octane est caractérisé par un nombre, «l'indice d'octane ».
} 
plus faible. Faute de traitement de craquage performant, on n'avait pas accès à l'époque aux gazoles et autres fuels lourds, comme sources potentielles de carburant. La seule technique existante était le craquage thermique qui mettait en œuvre des conditions opératoires difficiles (voir annexe 1).

Houdry démarre donc des essais d'orientation avec du nickel déposé sur du kaolin, essais «qui lui montrèrent qu'il y avait là une voie nouvelle possible $»^{20}$. Tout au long des années 1927 à 1930, Houdry va donc faire des recherches à Beauchamp dans ce domaine ${ }^{21}$, menant en parallèle des travaux sur le procédé avec le lignite et l'appui nécessaire à l'usine de Saint-Paulet. Ces travaux vont donner lieu à de nombreuses avancées scientifiques et techniques (voir annexe). Les brevets, à partir de décembre 1927, sont relatifs à des «procédés et dispositifs pour la fabrication de carburants par hydrogénation de masses gazeuses en présence de catalyseurs $»^{22}$, les gaz en question « provenant de la distillation ou de la pyrogénation de combustibles de moindre valeur $»$, incluant donc les produits issus de la distillation de pétrole brut.

Compte tenu de ces résultats, Houdry va rapidement prendre des contacts avec l'industrie du raffinage des pétroles, en vue de

\footnotetext{
${ }^{20}$ Joseph A., op.cit., note 3, p. 194.

21 J. Bureau, évoquant ses souvenirs quand aux relations entre Saint-Gobain et Houdry, mentionne les collaborateurs proches d'Houdry présents à Beauchamp, comme René Le Grain, administrateur de société, M. Lafond, directeur technique, et M. Joseph, École Polytechnique, "chargé de la documentation et des brevets », voir Bureau J, op. cit., note 17, p. 20.

22 Brevet français $n^{\circ} 659672$ du 20.12.1927 et son correspondant américain US1837963 (A).
} 
développer des collaborations ${ }^{23}$. Les premiers contacts datent de la deuxième moitié de l'année 1928. Joseph mentionne successivement ${ }^{24}$ :

-L'Anglo-Persian oil Company, ancêtre de British Petroleum, venue à Beauchamp en novembre 1928, qui aurait proposé de construire une unité de 1 t/j en Angleterre. Houdry aurait refusé en estimant «que le moment n'était pas venu de faire une pareille démonstration à l'étranger ». L'article de Fortune avance une autre raison : un manque d'engagement en terme de moyens de la part de la compagnie anglaise, qui n'aurait pas permis de développer ce nouveau procédé ${ }^{25}$.

- Des ingénieurs de la Batafsche Petroleum Maatschappij, ancêtre de la Shell, propriétaire d'une raffinerie qui allait être mise en route en France à Petit-Couronne l'année suivante en 1929, venus aussi expertiser les procédés Houdry en octobre 1928, et février 1929. Toutefois, après une étude de propriété industrielle et une proposition de collaboration début 1930, la société est revenue sur cette proposition et a décliné en avril 1930 l'offre de collaboration. En fait, la société hollandaise était en train de négocier avec l'I.G. Farben d'autres types de recherches.

\footnotetext{
${ }^{23}$ Après le redémarrage de la raffinerie de Courchelettes en 1927 (Société générale des huiles de pétrole, SGHP, filiale du groupe anglais L'Anglo-Persian), et la mise en service de celle de Petit-Couronne en 1929 (Shell), c'est, au début des années 1930 une succession de démarrage de nouvelles raffineries. Nous ne mentionnerons ici que celle de la Compagnie des Produits Chimiques et Raffineries de Berre, PCRB, filiale de Saint-Gobain, en 1931. Voir par exemple : Amphoux Marcel, "Une nouvelle industrie française: le raffinage du pétrole », Annales de géographie, 44/251, 509-533 (1935).

${ }^{24}$ Joseph A., op.cit., note 3, p. 199-201.

${ }^{25}$ Collectif, op.cit., note 14, p. 132.
} 
- La Compagnie française de raffinage (Pechelbronn) a fait faire et contrôler des tests de cracking en octobre 1929, mais tout en étant, elle aussi, intéressée, n'a pas donné suite.

-Des représentants de la Compagnie des produits chimiques et raffineries de Berre (PCRB), filiale de Saint-Gobain, sont venus assister à des tests en juillet $1930^{26}$, une visite qui donne lieu à un accord pour la construction à Berre d'une cellule de démonstration de $10 \mathrm{t} / \mathrm{j}^{27}$. Cette cellule ne verra jamais le jour, sans qu'on en sache réellement les causes; quelques mois plus tard, le 4 octobre 1930, Houdry signait un accord de collaboration avec la Vacuum oil Company...

À l'issue de tests effectués en septembre 1930, Harold Sheets, responsable des opérations internationales de la Vacuum oil vient rendre visite à Houdry début octobre et lui propose le marché suivant : si Houdry est capable de refaire aux États-Unis les tests faits à Beauchamp dans un délai de quinze jours, Sheets s'engage à financer le développement industriel du procédé. Houdry va relever le défi et part aux États-Unis à Paulsboro (raffinerie de la Vacuum) fin octobre accompagné d'un chef d'atelier et d'un ouvrier. Fin novembre « cette étape était franchie sans histoire $»^{28}$. Conformément à l'accord du 4 octobre, Vacuum oil entreprit la construction d'une unité de $10 \mathrm{t} / \mathrm{j}$

\footnotetext{
${ }^{26}$ Pourtant, peu de temps avant l'accord avec la PCRB (entre 1927 et 1930 ?) une délégation de Saint-Gobain vint à Beauchamp assister à une semaine d'essais, voir Bureau J., op. cit. note 17, p. 20. Le bilan de cette semaine d'essais ne fût toutefois pas très favorable au procédé de Houdry, et les représentants de Saint-Gobain conclurent au " rejet temporaire du projet » [de collaboration au développement de procédé ?]!

${ }^{27}$ Joseph A., op.cit., note 3, p. 200.

${ }^{28}$ Houdry E., op. cit., note 3, p. 203.
} 
conçue par Houdry, unité mise en route en mai 1931 et qui donnera les résultats attendus. Le 25 juillet 1931, la Houdry Process Corporation était fondée, au capital de 3,3 M\$ (1/3 vacuum, 2/3 Houdry et partenaires français), l'aventure américaine commençait.

\section{Les débuts de l'aventure américaine d'Houdry}

L'année 1931, celle du premier succès d'Houdry, voit la situation économique de l'industrie pétrolière se dégrader avec une baisse très importante du prix du brut. Ces difficultés vont être probablement à l'origine du regroupement cette même année, de la Vacuum avec la Standard Oil Company of New-york, pour donner naissance à la Socony-Vacuum le 31 juillet 1931. Deux ans plus tard en 1933, dans un climat économique toujours très maussade, et devant les réticences de la toute nouvelle société d'aller plus loin en matière industrielle, Houdry se décide à demander l'autorisation de contacter d'autres sociétés pétrolières pour l'industrialisation de son procédé :

"Houdry a fait le tour des compagnies pétrolières américaines. Il a proposé son procédé à pratiquement toutes les plus importantes. Quelques unes étaient moyennement intéressées. Certaines lui dirent carrément non. Mais avec Arthur Pew de la Sun Oil cela a été différent $»^{29}$.

Un accord entre Arthur Pew et Houdry va en effet être trouvé pour développer industriellement le procédé. Il semble bien que dès leur

\footnotetext{
${ }^{29}$ « Houdry made the rounds of U.S.Oils. He offered the process to virtually every important company. A few were midly interested. Some gave him a flat no. But with Arthur Pew of Sun Oil, it was different ». Voir Collectif, op.cit., note 14, p. 132.
} 
première rencontre, les deux hommes aient eu un très bon contact et développé une indéniable confiance mutuelle ${ }^{30}$.

Mais il faut aussi ajouter, que les propositions de Houdry « tombaient à point » en correspondant justement à un besoin de la $\operatorname{Sun}^{31}$. À cette période, à l'exception de la Sun, les compagnies augmentaient l'indice d'octane à l'aide de plomb tétraéthyle ${ }^{32}$. La Sun Oil était la seule à ne pas utiliser d'additifs «one grade fits all » (une composition convenant à tous), mais Arthur Pew, son président, malgré le soin pris à choisir et raffiner les meilleures coupes pétrolières possibles, était donc très intéressé à pouvoir commercialiser des essences toujours sans additifs mais plus performantes en terme d'indice d'octane. Houdry et son équipe déménagent donc de Paulsboro à la raffinerie Marcus Hook de la Sun Oil en juin 1933.

En l'espace de deux ans, Houdry, avec l'appui des ingénieurs de la Sun Oil (et même de la Socony-Vacuum qui restait intéressée au développement du procédé), va travailler à perfectionner et automatiser les séquences de régénération, la forme et la taille des catalyseurs. À l'issue de ces deux années, un accord intervint entre la Sun, la Socony-Vacuum et l'Houdry Process concernant le développement industriel du procédé dans les deux raffineries de Marcus Hook et de Paulsboro.

\footnotetext{
30 L'article de Fortune rapporte une commentaire d'Houdry à propos de cette entrevue : "The most beautiful day in my life », voir Collectif, op.cit. note 14, p. 132.

${ }^{31}$ Palucka T., op.cit., note 1, et Collectif, op.cit., note 14, p. 134.

${ }^{32}$ Le plomb tétraéthyle est un additif pour les essences qui permet d'améliorer les propriétés antidétonantes du carburant, et de ce fait leur indice d'octane (autour de 10 points).
} 
La première unité industrielle de craquage catalytique, «114 », a démarré le 31 mars 1937 à la Raffinerie Sun Oil de Marcus Hook (15 000 barils/jour). La charge était constituée par un fuel lourd. Le résultat fût à la hauteur de ce qui était attendu : 48\% d'essence à 81 d'indice d'octane et $52 \%$ de gazoles... À partir de là, le développement du procédé est allé très vite. Fin 1939, il y avait quinze unités de craquage en fonctionnement ou en construction, représentant une production de 212000 barils/jour. En 1944, c'était vingt-quatre unités produisant 330000 barils/jour. Par ailleurs, le craquage catalytique sera complété par une autre étape consistant à faire repasser les essences au craquage une deuxième fois afin d'augmenter encore l'indice d'octane ${ }^{33}$.

Grâce aux qualités de ces essences, il était possible d'obtenir des carburants pour l'aviation (100 d'indice d'octane), beaucoup mieux désulfurés et avec moitié moins d'additifs qu'avec les autres carburants.

\section{L'intérêt tardif du gouvernement français}

Début 1939, le ministre français des Travaux publics, Anatole de Monzie, demande à Houdry de «venir en France expliquer [son] procédé et nous permettre d'en tirer le meilleur parti national ${ }^{34}$. Lors d'une conférence/réunion le 7 février 1939 au ministère à Paris, Houdry apporte un projet d'installation de craquage catalytique permettant de produire de l'essence d'aviation, avec l'appui également de lettres des présidents de la Sun Oil et de la Socony-Vacuum,

\footnotetext{
${ }^{33}$ Oblad, A., op. cit., note 3, p. 74-75.

${ }^{34}$ Houdry E., op.cit., note 3, p. 206.
} 
s'engageant de leur côté à fournir de l'essence à la France si nécessaire. Malgré l'approbation des experts français présents, seule, toutefois, la PCRB accepte de construire dans sa raffinerie de Berre une unité de craquage catalytique ${ }^{35}$. Cette construction sera malheureusement suspendue en 1940 suite à l'occupation allemande.

L'appel du général de Gaulle du 18 juin 1940 va susciter un appui inconditionnel de la part d'Eugène Houdry. Avec d'autres français présents aux États-Unis ${ }^{36}$, il fonde le 26 août 1940 une association de soutien au général de Gaulle, France Forever, dont il devient le président et où Henri Laugier ${ }^{37}$, va s'illustrer à partir de $1941^{38}$. France Forever aura un rôle important en matière de

${ }^{35}$ La réunion organisée par le ministre semble correspondre davantage à une convocation de Houdry qu'à une simple invitation. Par ailleurs, elle ne semble pas susciter l'enthousiasme de la part des producteurs français puisqu'à part la PCRB aucune autre société n'a répondu positivement. Élément étonnant, car il y avait deux raffineries de la Vacuum Oil, en France à l'époque, l'une à Port Jérôme et l'autre à Frontignan en association avec la Compagnie industrielle des pétroles !

${ }^{36}$ Les autres cofondateurs étaient : Albert Simard, médecin à New York et Fred Hoffher, professeur à L'université de Columbia, avec l'appui actif, entre autres, de Paul Wertheimer.

${ }^{37}$ Henri Laugier (1888-1973), professeur de physiologie, sera à l'origine de diverses institutions scientifiques pendant le Front populaire. Chef de cabinet du ministre de l'Éducation nationale, il sera aussi le premier directeur du Service central de la recherche scientifique (ancêtre du CNRS), créé le 31 décembre 1936. Parti en exil (mi-1940) au moment de la débâcle, d'abord brièvement en Angleterre, il part au Canada où il est nommé professeur de physiologie à l'Université de Montréal début 1941. Il rejoint assez vite France Forever dont il sera un membre très actif. Il est appelé par le général de Gaulle à Alger au printemps 1943, où il sera nommé recteur de l'université. En 1946, il accédera au poste de secrétaire général adjoint de l'ONU, puis deviendra membre du conseil exécutif de l'UNESCO en 1952.

${ }^{38}$ À propos de France Forever et de Henri Laugier, voir Morelle Chantal, « Les années d'exil », in Henri Laugier en son siècle, Crémieux-Brilhac Jean-Louis et Picard Jean-François (dir.), Cahiers d'histoire pour la recherche, 3, (CNRS éditions 1995), réimpression sur http://www.histcnrs.fr/pdf/laugier-crh/morelle.pdf. Chantal Morelle décrit notamment la situation qui règne en Amérique du Nord entre les Français réfugiés, et les contacts étroits entre Henri Laugier et Eugène Houdry pendant les années 1941 et 1942. 
propagande et de soutien au général de Gaulle ${ }^{39}$ (meetings, films, articles, émission de radio...). La réaction du gouvernement de Vichy ne se fait pas attendre : par décret du 30 avril 1941, Houdry est déchu de sa nationalité française en même temps que vingt-huit autres personnalités (dont René Cassin, Henri Bernstein, André Kahn, Ève Curie...). Houdry demandera et obtiendra peu après (janvier 1942) la nationalité américaine ${ }^{40}$.

Quand au procédé de craquage catalytique, des améliorations importantes seront faites dès le début des années 1940. Le procédé initial consistait en une batterie de réacteurs en parallèle dont certains fonctionnaient en craquage pendant que d'autres étaient en régénération. En mai 1942, un procédé continu « à lit fluide ${ }^{41}$ », FCC (Fluid Cracking Catalytic), est mis en service par la Standard Oil of New Jersey à Bâton Rouge en Louisiane. En 1943, les ingénieurs de la Socony-Vacuum développeront eux aussi un procédé continu «à lit mobile » d'un principe un peu différent, le TCC (thermofor cracking catalytic).

\footnotetext{
${ }^{39}$ Nous ne savons pas exactement quand et pourquoi Houdry laissera la présidence de France Forever (1943 ?), peut-être suite au départ d'Henri Laugier avec lequel il semblait proche, peut-être à cause du contexte de l'époque concernant les Français aux États-Unis, où la division entre associations de soutien au général de Gaulle s'était accentuée, et ne lui convenait plus?

${ }^{40}$ Il n'est pas certain qu'Houdry ait vraiment apprécié la rencontre à Paris avec le ministre de Monzie en 1939. Par ailleurs il était devenu une personnalité marquante et reconnue aux États-Unis. Enfin, c'est aux États-Unis qu'il avait eu les moyens de développer ses idées, voir à ce sujet McEvoy James E., "Citizen Houdry », Chemtech, 26, 6-10 (Feb. 1996), voir p. 9.

${ }^{41}$ Le catalyseur n'est plus sous forme de grain, mais sous forme d'une poudre (taille moyenne inférieure à 50 microns). Dans les conditions convenables de vitesse des gaz (vapeurs d'hydrocarbures ou air), le catalyseur peut être « fluidisé », c'est-à-dire en suspension stable dans le gaz et transporté du réacteur au régénérateur et retour.
} 
À la fin de la guerre la production des unités de «type Houdry » était supérieure à 900000 barils/jour. En 1942-1943, à l'appui de tout son savoir-faire en matière de développement de procédé, la Houdry Process Co sera chargée de mettre au point un procédé de production de butadiène par déshydrogénation du butane, ce qu'elle fera avec succès. En 1948, Houdry prend un peu de champ avec le craquage et demande à la Houdry Process Co de « le libérer d'une grande partie de ses obligations (...) pour [lui] permettre d'explorer le champ d'application des catalyseurs d'oxydation $»^{42}$.

Dans un nouveau contexte, il fonde une autre société «OxyCatalyst Corporation », et va mettre au point un système catalytique, l' « oxycat», pour la combustion de gaz pauvres à une température basse, essentiellement destiné au traitement des gaz effluents des chaudières. Lors du choix et de l'étude des catalyseurs, Houdry mit en évidence la présence d'hydrocarbures cancérigènes dans ces gaz. Il décide alors d'aborder un autre cas, celui des gaz d'échappement des moteurs à combustion interne et confirme la présence de microparticules $(0,01 \text { micron })^{43}$ : « J'ai pensé qu'il pouvait y avoir une relation directe entre cette constatation et l'augmentation des cas de cancer $\gg$.

À partir de 1950 il va s'attacher à étudier des catalyseurs permettant d'obtenir une post-combustion totale des gaz. À cet égard il sera un des pionniers majeurs dans le développement des «pots catalytiques $\gg{ }^{44}$. Cela va être son ultime quête, à savoir lutter contre la

\footnotetext{
${ }^{42}$ Houdry E., op.cit., note 3, p. 210.

${ }^{43}$ Houdry E., op.cit., note 3, p. 211.

${ }^{44}$ Selon George E. Lester, Houdry serait l'auteur d'un brevet visionnaire relatif aux caractéristiques de ce que devraient être les «pots catalytiques» en 1956 : US
} 
pollution de l'air. Le premier congrès international sur la catalyse a lieu aux États-Unis à Philadelphie à l'automne 1956, où au cours d'une conférence plénière, Eugène Houdry détaille les grandes étapes de ses innovations, notamment dans la lutte contre la pollution de l'air ${ }^{45}$.

Un peu plus tôt la même année il avait fait une visite en France et, à cette occasion, donné une conférence avec son adjoint A. Joseph devant des représentants de l'Association française des techniciens du pétrole le 18 avril $^{46}$. Il revient à Paris en 1960 lors du deuxième Congrès international sur la catalyse, où à nouveau, il donne une conférence plénière, cette fois en français ${ }^{47}$ ! Il décéde peu de temps après, le 18 juillet 1962, à Upper Darby (Pennsylvanie).

\section{Conclusion}

Houdry, c'est 220 brevets, à l'image d'un « engagement» inlassable en matière de recherche et développement industriels. Dans un grand nombre de domaines, ces travaux seront à l'origine d'avancées marquantes en terme de procédés, et beaucoup considèrent que sans le craquage catalytique, qui permit d'intensifier la fourniture d'essence à haut indice d'octane pour l'aviation, l'issue de la guerre

Patent $\mathrm{n}^{\circ} 2742437 \mathrm{du}$ 7.04.1956. Voir Lester G.E., « The development of automotive exhaust catalysts» in Davis Burtron H. et Hettinger William P. (eds), Heterogeneous Catalysis, op. cit. note 3, p. 415-433, voir p. 418.

${ }^{45}$ Houdry E., «Practical analysis and its impact on our génération », Advances in Catalysis, 9, 499-509 (1957).

${ }^{46}$ Houdry E., op.cit., note 3, et Joseph A., op.cit., note 3.

${ }^{47}$ Houdry E., « Développements et tendances de la catalyse industrielle », Actes $d u$ deuxième Congrès international de catalyse, Paris, 1960, Éditions Technip, Paris, 1961, p. 149-158. Conférence plénière. 
n'aurait peut être pas été la même. Il a reçu de nombreuses récompenses académiques prestigieuses : la «Potts Medal of the Franklin Institute » (1948), la « Perkin Medal of the American Section of the Society of Chemical Industry » (1948), la « Murphree Award in Industrial and Engineering Chemistry of the American Chemical Society » et a été élu à titre posthume au « National Inventors Hall of Fame » américain en 1990.

$\mathrm{Si}$ son aventure américaine a donné lieu a beaucoup d'évocations, son parcours en France et ses relations françaises sont un peu moins connues, entre autres la nature et l'origine des moyens économiques français qui l'ont soutenu dans les années 1920, les circonstances de son entrevue avec le ministre de Monzie en 1939, ses relations avec les «pétroliers » français, enfin son positionnement et son rôle dans France Forever pendant la guerre au milieu des expatriés français. Cela fera l'objet d'un travail complémentaire. 


\section{Annexe 1 : \\ Demande d'autorisation d'Houdry pour Beauchamp, p. 1}

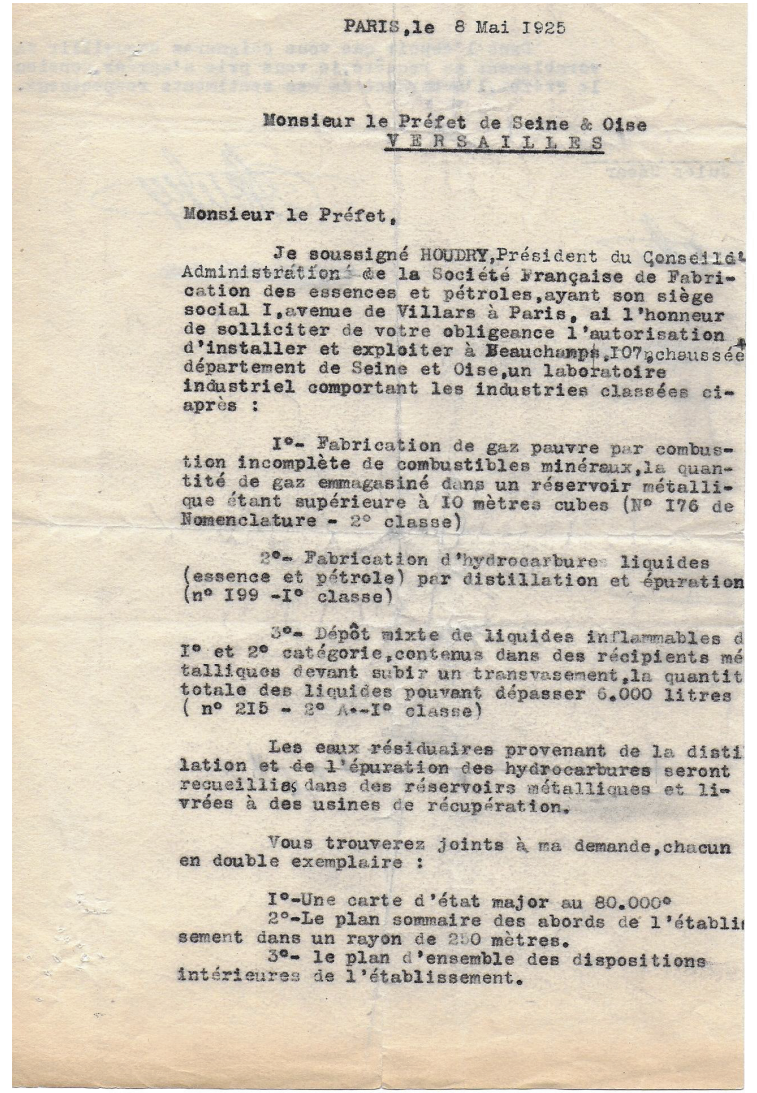

Annexe 2 : Aspects techniques

Par distillation d'un brut pétrolier, on obtient très schématiquement et selon son origine (entre parenthèses le nombre de carbones des chaines hydrocarbonées) : environ $1 \%$ de gaz (C1-C4); 10 à $25 \%$ d'essences $(\mathrm{C} 5-\mathrm{C} 10) ; 5$ à $10 \%$ de kérosènes $(\mathrm{C} 10-\mathrm{C} 13) ; 30$ à $50 \%$ de gazoles $(\mathrm{C} 13-\mathrm{C} 25) ; 20$ à $40 \%$ de résidus lourds $(>\mathrm{C} 25)$. 
Le craquage des fractions lourdes ${ }^{48}$ (gazoles, certains résidus lourds) consiste en la scission d'hydrocarbures aliphatiques saturés en paraffines et oléfines (par exemple : C30 en une paraffine C8 et une oléfine C22. D'autres réactions de craquage (craquage secondaire) de ces types de produits ont ensuite lieu pour donner divers hydrocarbures plus légers. D'autres processus peuvent interférer en fonction des conditions opératoires (autres déshydrogénations, isomérisations, oligomérisations...).

Le principe du craquage thermique était connu de longue date, mais n'avait été mis en œuvre industriellement qu'en 1913, avec le procédé Burton. C'était un procédé discontinu, à haute température $\left(400^{\circ} \mathrm{C}\right)$ et à pression modérée $(5$ bars $)$, avec des problèmes de nettoyage importants (décokages fréquents). Des améliorations furent faites dans les années 1920 avec le développement de plusieurs procédés continus (entre autres, Holmes-Manley process, Dubbs, «tube and tank », Cross...), mais toujours avec des problèmes de cokage en raison des pressions alors mises en œuvre (30 à 70 bars) et des températures très élevées (autour de $500^{\circ} \mathrm{C}$ ). Dans tous les cas les schémas de procédé étaient compliqués et surtout les essences issues du craquage, dépendantes de l'origine du brut, étaient de médiocre qualité en terme d'indice d'octane ${ }^{49}$.

Le craquage catalytique apparut dès 1915 avec l'utilisation d'un catalyseur, le chlorure d'aluminium, qui permettait d'abaisser

\footnotetext{
${ }^{48}$ Voir par exemple Decroocq Daniel, Bulle R., Chatila S., Franck J.P. et Jacquin Y., Le craquage catalytique des coupes lourdes, Éditions Technip, Paris, 1978.

${ }^{49}$ Voir par exemple, en ce qui concerne la comparaison technico-économique des procédés de craquage : Enos John Lawrence, Petroleum progress and profits : a history of process innovation, MIT Press, Cambridge, Mass, 1962.
} 
notablement la pression (pression atmosphérique) et la température $\left(<300^{\circ} \mathrm{C}\right)$. Le procédé fût un échec en raison du prix élevé du catalyseur et des problèmes de corrosion, inhérents à la présence d'acide chlorhydrique. Le choix d'un autre catalyseur était donc un paramètre fondamental pour le développement de ce type de procédé. Decroocq $^{50}$ rappelle que Paul Sabatier et Alphonse Mailhe avaient déposé un brevet la même année (1915) recommandant l'utilisation de catalyseurs à base d'oxydes métalliques pour le craquage des hydrocarbures. Houdry a souvent évoqué le nom de Sabatier comme une de ses sources d'inspiration ${ }^{51}$.

Les innovations de Houdry vont se situer à 2 niveaux : (i) le développement de procédé, en particulier au niveau de la régénération des catalyseurs, (ii) la recherche de catalyseurs (phase active et support) aussi bien pour le craquage que pour la désulfuration.

- (i). En matière d'épuration du soufre présent dans les lignites, cette régénération était essentielle pour éviter l'empoisonnement des catalyseurs de craquage. Très vite, l'utilisation des «masses de Laming » fût abandonnée au profit d'une fixation des dérivés du soufre (hydrogène sulfuré, sulfures organiques) sur un solide à base de $\mathrm{Ni} /$ kaolin. Le contrôle de la température était rigoureux, à un niveau ni trop élevé pour éviter de faire du craquage à ce stade, ou à l'inverse ni trop bas pour éviter de condenser les vapeurs de goudrons. Par ailleurs il fallait aussi éviter la transformation du nickel sous forme de sulfates basiques ! Le kaolin fût abandonné en 1927, et remplacé par un

\footnotetext{
${ }^{50}$ Decroocq et al., op. cit., note 48, p. 14.

${ }^{51}$ Houdry E., op. cit., note 45, p. 501.
} 
support à base de terre de Mussidan (48\% silice, 37\% d'alumine), d'un liant constitué de mazout lourd et de sciure de bois (sapin).

Que ce soit pour la régénération des solides de désulfuration, ou le décokage des catalyseurs de craquage, la régénération s'effectuait par soufflage d'air en alternance (un appareil en opération, un appareil en régénération).

Pour éviter des élévations de température trop élevées, Houdry conçut un régénérateur de forme annulaire, où le catalyseur était disposé en couches horizontales séparées par des chambres vides pour l'alimentation latérale de l'air ou l'évacuation des fumées. Finalement en juillet 1930, Houdry arriva à la solution de l'immersion des tubes échangeurs dans la masse de catalyseur, tubes alimentés en eau et ultérieurement en sels métalliques fondus.

- (ii). Et pour les premières études de craquage de gazoles (1927), Houdry mit d'abord en œuvre un catalyseur constitué de $\mathrm{Ni} /$ kaolin (le même que pour la désulfuration) :

"L'appareillage était très simple; il comportait uniquement un vaporiseur, une caisse contenant les catalyseurs et un déflegmateur ${ }^{52}$ permettant de séparer les lourds pour les recycler. Aucune régénération n'avait été prévue. Les produits obtenus étaient de qualité médiocre et les pertes en gaz et en coke étaient élevées. La proportion de légers dans les produits craqués allant rapidement en diminuant avec la durée du traitement, l'appareil fut modifié pour munir la caisse de cracking d'un dispositif de régénération in situ par soufflage d'air. L'expérience montra que les périodes de régénération étaient fréquentes et longues $»^{53}$.

\footnotetext{
${ }^{52}$ C'est-à-dire un distillateur.

${ }^{53}$ Joseph A., op.cit., note 3, p. 194.
} 
Houdry va alors entreprendre une étude approfondie du catalyseur, et en particulier du support, le kaolin manquant de porosité et se prêtant mal à la régénération. Il s'orienta alors vers des «terres à foulon », traitées à l'acide sulfurique pour enlever un maximum d'impuretés, auxquelles il ajoutait quand même un peu d'argile. Mais cela n'apporta pas d'améliorations significatives. Finalement au début de 1929, Houdry essaya d'utiliser un support sans métal. Les meilleurs résultats furent obtenus avec une «terre de San Diego » (74\% de silice, 13\% d'alumine), où il était possible de réduire la quantité de liant nécessaire à la préparation des grains de catalyseur. Il adopta définitivement ce type de catalyseur : 70 à $80 \%$ de silice, 10 à $20 \%$ d'alumine, impuretés inférieures à $10 \%$. 